\title{
Parameterization of the optical constants of polydopamine films for spectroscopic ellipsometry studies
}

Qie, Runtian; Moghaddam, Saeed Zajforoushan; Thormann, Esben

Published in:

Physical Chemistry Chemical Physics

Link to article, DOI:

10.1039/d0cp04796a

Publication date:

2021

Document Version

Peer reviewed version

Link back to DTU Orbit

Citation (APA):

Qie, R., Moghaddam, S. Z., \& Thormann, E. (2021). Parameterization of the optical constants of polydopamine films for spectroscopic ellipsometry studies. Physical Chemistry Chemical Physics, 23, 5516-5526.

https://doi.org/10.1039/d0cp04796a

\section{General rights}

Copyright and moral rights for the publications made accessible in the public portal are retained by the authors and/or other copyright owners and it is a condition of accessing publications that users recognise and abide by the legal requirements associated with these rights.

- Users may download and print one copy of any publication from the public portal for the purpose of private study or research.

- You may not further distribute the material or use it for any profit-making activity or commercial gain

- You may freely distribute the URL identifying the publication in the public portal

If you believe that this document breaches copyright please contact us providing details, and we will remove access to the work immediately and investigate your claim. 


\section{Parameterization of the Optical Constants of Polydopamine Films for Spectroscopic Ellipsometry Studies}

aReceived 00th January 20xx Accepted 00th January 20xx

DOI: $10.1039 / x 0 x \times 00000 x$

\begin{abstract}
Runtian Qie, Saeed Zajforoushan Moghaddam, and Esben Thormann*
Bio-inspired polydopamine coatings offer vast possibilities for surface modification of materials. The thickness of such nanometric coatings is usually estimated based on ellipsometry measurements. However, the complex light-absorbing nature of polydopamine is often overlooked when analyzing such data, which can result in inaccurate estimations of the coating thickness as well as the optical properties. In this study, we prepared and characterized three polydopamine coatings where the film thickness and surface roughness are systematically varied. For each case, we developed suitable optical models and showed how an improper optical model can provide inaccurate estimates of the coating properties. AFM height profiles were obtained from scratched areas of each sample to verify the thickness values estimated by ellipsometry. The results confirm that polydopamine coatings, depending on the oxidation conditions, can possess different structural and optical properties, and thus require unique optical models for the ellipsometry analysis.
\end{abstract}

\section{Introduction}

Polydopamine (PDA) has drawn enormous attention as a versatile coating material that can readily form nanometric thin films on different materials. ${ }^{1-3}$ Besides, PDA coatings can be codeposited or post-functionalized with different molecules providing a wealth of surface modification possibilities. ${ }^{4-8}$ Owing to these advantages, there has been a growing research effort devoted to the fabrication and characterization of PDA coatings during the past decade. Spectroscopic ellipsometry, as a powerful tool to estimate the thickness of organic and inorganic thin-films, is often employed to characterize PDA coatings. ${ }^{9-18}$ Ellipsometry is a non-destructive optical method that measures the changes in the polarization state of the light reflected from a thin-film on a substrate. ${ }^{19,20}$ The film thickness is indirectly estimated from the experimental data by constructing a proper model, which represents the optical behavior of the material. For materials with known optical constants, the film thickness is simply obtained as the only "free" parameter in the model. Contrarily, materials with unknown optical constants are challenging because the thickness as well as the optical constants are free model parameters. This brings up two issues: (i) choosing the appropriate dispersion equation that can describe the optical behavior of the material, (ii) the possibility of overparameterization and correlation between the free model parameters. Hence, samples with unknown optical constants

Department of Chemistry, Technical University of Denmark, 2800 Kgs. Lyngby, Denmark.

Electronic Supplementary Information (ESI) available: [additional XPS data, water contact angle data, detailed ellipsometry modeling data and sensitivity analysis, optical functions estimated from modeling, ellipsometry data of $24 \mathrm{~h}$ deposition PDA ]. should be subjected to rigorous analysis to ensure the reliability of the modeling outcome.

Polydopamine coatings comprise a complex mixture of different aromatic compounds. ${ }^{21,22}$ The covalent coupling of dopamine occurs through the oxidation of the catechol group to quinone, followed by a cyclization reaction generating indole, as well as other oxidation and reorganization reactions forming several monomeric and oligomeric products. ${ }^{23}$ These compounds aggregate through various supramolecular interactions and produce polydopamine particles in the solution and thin-films on the immersed substrates. Given such chemical heterogeneity, polydopamine possesses a rather complex optical dispersion with multiple absorption features over a broad range of wavelength from UV to NIR regions. ${ }^{24-27}$ Besides, the oxidation conditions affect the chemical composition and structure of polydopamine films. ${ }^{23}$ Therefore, the optical properties of PDA can also vary depending on the reaction conditions, meaning that obtaining "tabulated" optical constants for PDA is infeasible. Such complex optical behavior of PDA necessitates a careful modeling approach to analyse the ellipsometric data of PDA films. Moreover, the intrinsic granular, porous, and rough nature of PDA films is another complication that must be considered. Such careful analysis and report of the ellipsometric data of PDA films is often overlooked. Many studies still rely on the Cauchy model, which is only valid for transparent materials with no or negligible light absorption. Besides, many studies report merely the estimated thickness without unfolding the modeling procedure and assessing the quality of the modeling data.

In this study, we prepared and characterized three PDA samples through different deposition times, i.e., 2 h, 6 h, and 12 h. By doing so, we obtained PDA films of systematically varied thickness and surface roughness; thus, we could optimize the modeling approach for each case. The estimated thickness for 
each sample was also verified by parallel AFM measurements on scratched films. It is shown that PDA samples obtained from different deposition times need unique optical models, depending on their structural and optical characteristics. Moreover, the misuse of the optical models provides significantly overestimated optical constants and underestimated thickness values. Hence, even if the optical constants of PDA are not of interest, the estimated film thickness will be inaccurate unless an appropriate optical model is utilized.

\section{Methods}

\subsection{Experimental Section}

Dopamine hydrochloride $\quad$ (DA) and from Sigma-Aldrich. DA was dissolved in tris-buffer solution $(\mathrm{pH}$ $=8.5,50 \mathrm{mM}$ ) and silicon wafers (WaferNet, San Jose, USA) were immersed into the DA solution $\left(2 \mathrm{mg} \cdot \mathrm{mL}^{-1}\right)$ for 2,6 , and 12 $h$. The DA solution was exchanged with a fresh solution every 2 $h$. Between each exchange step, the wafers were sonicated in fresh tris-buffer, then dried with compressed air. This procedure ensures a smoother surface with less aggregates, ${ }^{9,28}$ which can improve the quality of the ellipsometric data. Similar samples were prepared on glass slides for transmission measurements.

To analyse the chemical composition of the PDA films, X-ray photoelectron spectroscopy (XPS) measurements were conducted using a Thermo Scientific K-Alpha X-ray photoelectron spectrometer equipped with a hemispherical analyser and an $\mathrm{Al} \mathrm{K \alpha}$ micro-focused monochromator. Peak analysis was conducted using Avantage software. Surface charging was corrected with carbon C1s peak at $284.8 \mathrm{eV}$. The "smart" background and mixed Lorentzian (30\%)/Gaussian (70\%) functions were used. An initial value of $1.7 \mathrm{eV}$ was used for full width at half maximum (FWHM) of all peaks, which could vary between 1.5-1.9 eV. ${ }^{29}$ Spectroscopic ellipsometry measurements (M-2000U, J.A. Woollam Co., Inc.) were performed in the wavelength range of $245-1000 \mathrm{~nm}$ at 5 different angles of incidence $\left(50,55,60,65,70^{\circ}\right)$. Transmission measurements $\left(90^{\circ}\right)$ were conducted on bare and PDA-coated glass slides. For each PDA sample, a total number of 15 measuring points (five randomly selected areas on three specimens) were obtained and the median representative data are presented. The instrument software (CompleteEASE, J.A. Woollam Co., Inc.) was used for data modeling and analysis. Atomic force microscope (AFM, NanoWizard 3, JPK Instruments $A G$, Berlin, Germany) operating in tapping mode in air was used to estimate the thickness of the PDA coatings. The coatings were carefully scratched using a tweezer and AFM images were collected over the scratched area. The images were collected using a standard tapping mode cantilever (HQ: NSC5/AL BS, tip radius $\sim 8 \mathrm{~nm}, \mathrm{k} \sim 40 \mathrm{~N} . \mathrm{m}^{-1}$; Mikromasch, Germany) with a scan rate of $0.1 \mathrm{~Hz}$ and pixel resolution of $256 \times 256$. The thickness was then estimated from the cross-section height profiles (three replicas) using the standard software of the instrument (JPK SPM Data Processing).

\subsection{Theory and Modeling Procedure}

In an ellipsometry experiment, p-polarized (parallel to the plane of incidence) and s-polarized (perpendicular to the plane of incidence) light waves are irradiated on a flat sample. The interaction of light with the material gives rise to a variation in the polarization state of the reflected light, which is quantified in terms of ellipsometric $\psi$ and $\Delta$. The former represents the amplitude ratio and the latter indicates the phase difference between $\mathrm{p}$ - and s-polarizations, respectively. The measured $\psi$ and $\Delta$ values are related to the sample properties through the complex reflectance function $(\rho) .^{30}$

$$
\tan \Psi e^{i \Delta}=\rho\left(N_{a}, N_{s}, N_{1} \ldots, N_{j}, d_{1}, \ldots, d_{j}, \theta_{0}\right)
$$

The change in polarization state is then related to the angle of incidence $\left(\theta_{0}\right)$, the thicknesses $\left(d_{j}\right)$ of the layers, as well as the complex optical constants of medium $\left(\mathrm{N}_{\mathrm{a}}\right)$, substrate $\left(\mathrm{N}_{\mathrm{s}}\right)$, and the layers $\left(\mathrm{N}_{\mathrm{j}}\right)$. The optical constants of a material describe how it interacts with the light, which can be characterized using the complex refractive index $(\mathrm{N})$ or the complex dielectric function $(\varepsilon) .{ }^{31}$

$$
\begin{aligned}
& N=n \pm i k \\
& \varepsilon=\varepsilon_{1} \pm i \varepsilon_{2}
\end{aligned}
$$

The complex refractive index specifies how light-matter interaction modifies the light properties in terms of phase velocity, direction of propagation, and intensity loss. Contrarily, the complex dielectric function characterizes how light-matter interaction affects the material in terms of dielectric polarization. These two parameters are then related to each other by:

$$
\begin{gathered}
\varepsilon=N^{2} \\
\varepsilon_{1}=n^{2}-k^{2} \\
\varepsilon_{2}=2 n k
\end{gathered}
$$

Since the optical constants of the material depend on the light wavelength $(\lambda), N(\lambda)$ and $\varepsilon(\lambda)$ are recognized as the optical dispersions of the material. Alternatively, the optical constants can be described as a function of photon energy, which is related to wavelength through:

$$
E(\mathrm{eV})=\frac{1240}{\lambda(n m)}
$$

After collecting the measured $\psi(\lambda)$ and $\Delta(\lambda)$, one must construct an appropriate optical model that represents the nominal structure of the multilayer sample comprising information on thickness and optical constants of all the constituents. Next, the Fresnel's equations are used to calculate $\psi(\lambda)$ and $\Delta(\lambda)$ for the constructed optical model and the best match (with the smallest mean squared error (MSE)) between the measured and modelled values is obtained through regression. 
The PDA-coated silica wafer is herein represented with a threelayered optical model. The bottom-most component is an optically thick silicon (c-Si) substrate, i.e., characterized by its tabulated optical constants. A thermally grown oxide layer $\left(\mathrm{SiO}_{2}\right)$ is found on top of the substrate, which is characterized by its thickness and optical constants. The values of optical constants from the software library are used, while the thickness ( $100 \mathrm{~nm}$ ) of the oxide layer was measured prior to PDA deposition and then was fixed in the model. The topmost layer represents the PDA coating characterized by the thickness and optical constants. Since the optical constants of PDA are unknown, an appropriate model is then required to describe its dielectric function and optical behavior. ${ }^{32-36}$

The Cauchy relation is an empirical equation that is commonly used to describe the optical dispersion of transparent dielectrics and organic materials.

$$
\begin{gathered}
n(E)=A+B E^{2}+C E^{4} \\
k(E)=0
\end{gathered}
$$

Where $A$ adjusts the amplitude of the index of refraction, while $B$ and $C$ account for the curvature of the dispersion. The Cauchy relation can be used only if the material displays a "normal" dispersion, i.e., $\varepsilon_{1}$ and $\mathrm{n}$ values increase towards larger photon energy values (or shorter wavelengths) and light absorption is negligible $\left(\varepsilon_{2}\right.$ and $\left.\mathrm{k} \sim 0\right)$ over the investigated wavelength range. Generally, there is a possibility of correlation between these parameters (specifically between B and C), so extra care is needed to avoid overparameterization and physically implausible dispersion shapes. In case of minor light absorption, one can modify the Cauchy relation by adding the Urbach absorption term:

$$
k(E)=\alpha e^{\beta\left(E-E_{b}\right)}
$$

The extinction coefficient is then characterized by the amplitude $(\alpha)$, the exponent factor $(\beta)$, and the band-edge energy $\left(E_{b}\right)$, which together produce a small exponentiallydecaying extinction coefficient. This term is useful if the absorption is sufficiently small $(k<0.01)$ and the index of refraction still shows a normal dispersion, e.g., weak light absorption in the UV range.

For samples with a strong light absorption over a broad range of wavelengths, the Urbach term cannot properly describe the optical behavior. Such materials are usually characterized by an "anomalous" dispersion, where $\varepsilon_{2}$ displays multiple peaks, at which $\varepsilon_{1}$ shows a negative slope (decreases with increasing photon energy). The alternative method is then to parameterize the optical dispersion using a mathematical spline function, specifically when information on the optical constants of the material is limited. The spline function splits the entire spectrum into defined intervals, over which the optical dispersion curve is described using a simple polynomial function. The interval size can be tuned by the number of "nodes" defined along the energy (wavelength) axis. A basisspline (B-spline) function sums all the individual basis functions to construct the final optical dispersion rather than joining the curves. Accordingly, a B-spline function is defined as a recursive set of single polynomial splines. ${ }^{37,38}$

$$
\begin{gathered}
B_{i}^{0}(x)=\left\{\begin{array}{cc}
1 & t_{i} \ll x \ll t_{i+1} \\
0 & \text { otherwise }
\end{array}\right. \\
B_{i}^{k}(x)=\left(\frac{x-t_{i}}{t_{i+k}-t_{i}}\right) B_{i}^{k-1}(x)+\left(\frac{t_{i+k+1}-x}{t_{i+k+1}-t_{i+1}}\right) B_{i+1}^{k-1}(x)
\end{gathered}
$$

Where $\mathrm{k}$ represents the degree of the spline (usually $3^{\text {rd }}$-degree polynomial function, including this study), $i$ is the index (number) for the nodes $\left(t_{i}\right)$ over which the polynomial functions connect. The multiple basis functions (spline components) are then summed at each node to produce the overall shape of the dielectric function.

$$
S(x)=\sum_{i=1}^{n} c_{i} B_{i}^{k}(x)
$$

Where $c_{i}$ are the coefficients that adjust the amplitude of each spline component. One can decrease the spacing (interval size) between the nodes to gain more control over the shape of the B-spline curve. Nevertheless, while increasing the number of nodes might seem to better describe the shape of the dispersion, it will usually lead to unrealistic dispersions in which only the noise is described better. On the other hand, the larger the number of free parameters the higher the chance of correlation between the parameters will be. Hence, generally one should begin with an estimated lowest number of nodes and systematically increase the number of nodes. The number of nodes is directly related to the number of free parameters; therefore, increasing the number of nodes is acceptable only if a marked improvement in the modeling quality is achieved, otherwise, the lower number of nodes would be desired. ${ }^{39}$ Furthermore, B-spline curves render a "convex hull" property, i.e., the summed function cannot exceed the highest or lowest node amplitudes, which allows the $B$-spline to remain positive if all spline coefficients are $\geq 0$, thus avoiding non-physical solutions with negative $\varepsilon_{2}$ values. However, since B-spline is a purely mathematical parameterization of the optical dispersion, it cannot ensure the physical correlation between $\varepsilon_{1}$ and $\varepsilon_{2}$. To address this problem, Kramers-Kronig (KK) relations are used together with the B-spline function. Accordingly, the real and imaginary parts of the dielectric function are not independent, so $\varepsilon_{1}$ can be obtained by the KK-transform of $\varepsilon_{2}$. Hence, the general approach is to parametrize $\varepsilon_{2}$ using the B-spline function and then $\varepsilon_{1}$ is calculated using the Kramers-Kronig causality relation: ${ }^{37}$

$$
\varepsilon_{1}(E)=1+\frac{2}{\pi} P \int_{0}^{\infty} \frac{E^{\prime} \varepsilon_{2}\left(E^{\prime}\right)}{E^{\prime 2}-E^{2}} d E^{\prime}
$$

Where $P$ is the principal part of the integral. When parametrizing both $\varepsilon_{1}$ and $\varepsilon_{2}$ using B-splines, the number of free parameters is approximately twice the number of nodes. On the other hand, when parameterizing $\varepsilon_{2}$ only and then calculating $\varepsilon_{1}$ from the KK relation, the number of fitting parameters is reduced by almost half. In addition, it is ensured that the dielectric function adopts a physically plausible shape, i.e., light 

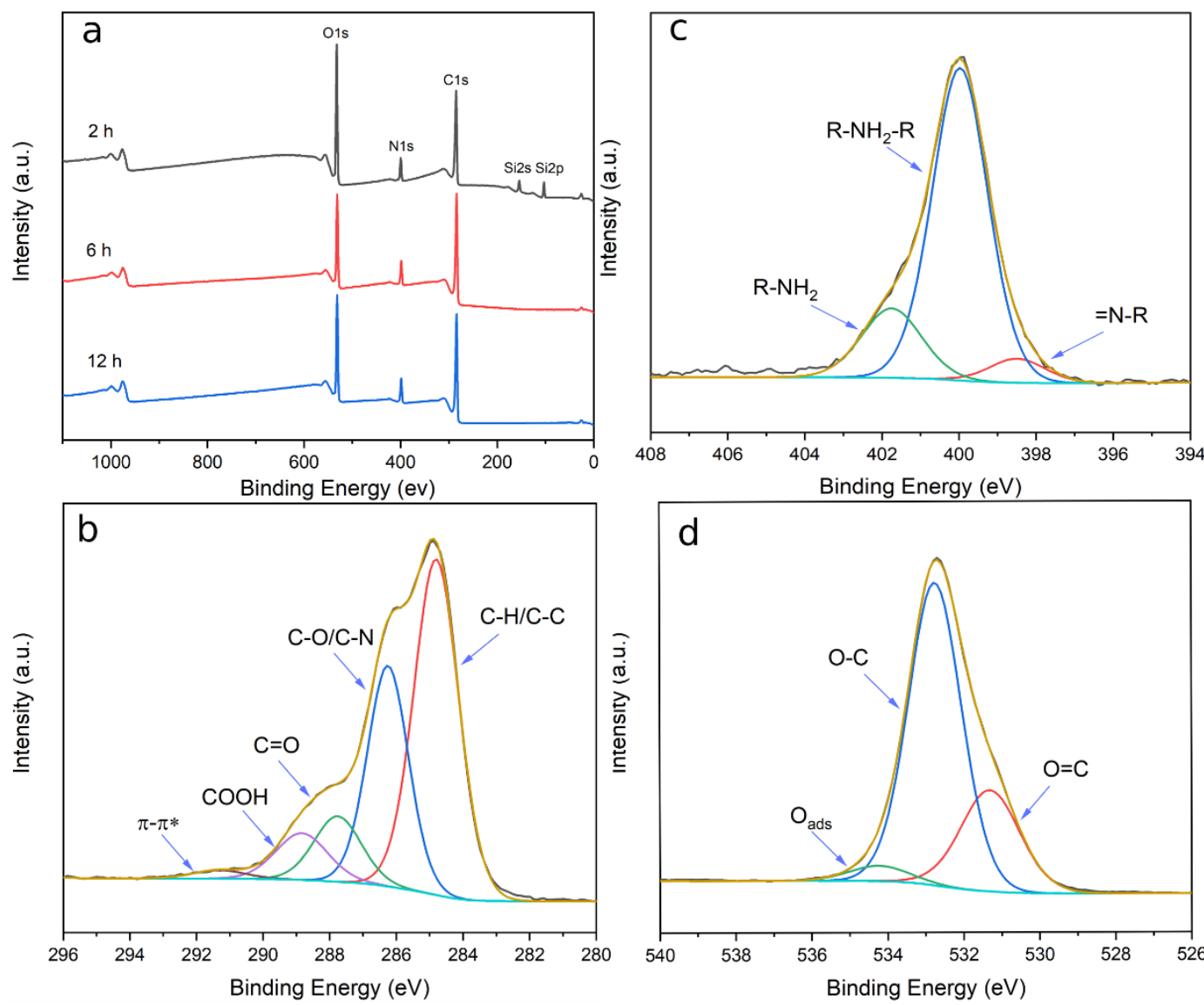

Figure 1 Surface characteristics of PDA films. (a) XPS spectra of PDA films of varying deposition times; high-resolution XPS spectra of (b) C 1s, (c) N 1s and (d) O 1s regions of PDA 2 h deposition

absorption causes anomalous dispersion (absorption bumps/peaks in $\varepsilon_{2}$ produce wiggles in $\varepsilon_{1}$ ) and the larger the area under $\varepsilon_{2}$, the larger the effect on $\varepsilon_{1}$.

The surface roughness can be modelled as a mixed material-air layer, whose complex dielectric function is described by an effective medium approximation (EMA) such as the Bruggeman's EMA: ${ }^{40,41}$

$$
f_{A} \frac{\varepsilon_{A}-\varepsilon_{E M A}}{\varepsilon_{A}+2 \varepsilon_{E M A}}+f_{B} \frac{\varepsilon_{B}-\varepsilon_{E M A}}{\varepsilon_{B}+2 \varepsilon_{E M A}}=0
$$

Table 1 Atomic composition of the PDA coatings determined by XPS

\begin{tabular}{|c|c|c|c|c|}
\hline $\begin{array}{c}\text { Deposition } \\
\text { time }\end{array}$ & C1s & O1s & N1s & Si2p \\
\hline $2 \mathrm{~h}$ & 57.8 & 28.1 & 7.7 & 6.4 \\
\hline $6 \mathrm{~h}$ & 69.3 & 22.1 & 8.6 & - \\
\hline $12 \mathrm{~h}$ & 67.3 & 24.2 & 8.5 & - \\
\hline
\end{tabular}

\section{Results}

PDA films are prepared through different deposition times, i.e., 2,6 , and $12 \mathrm{~h}$. Figure 1 (panel a) and Table 1 summarize the XPS chemical analysis and atomic composition of the coatings. The signals of $\mathrm{C}, \mathrm{N}$, and $\mathrm{O}$ atoms are found in all the samples, implying successful deposition of PDA coatings. The signal of the silica substrate (Si $2 p)$ is found for PDA $2 \mathrm{~h}$ sample, whereas it is undetected for PDA $6 \mathrm{~h}$ and $12 \mathrm{~h}$ due to the larger thickness of these samples. The calculated N/C ratio of the PDA 2, 6, and $12 \mathrm{~h}$ films is $0.131,0,119$ and 0.121 , respectively. It can be seen that $\mathrm{N} / \mathrm{C}$ ratio of PDA films obtained with different deposition times are $\approx 0.12$, which is comparable to the stoichiometric atomic

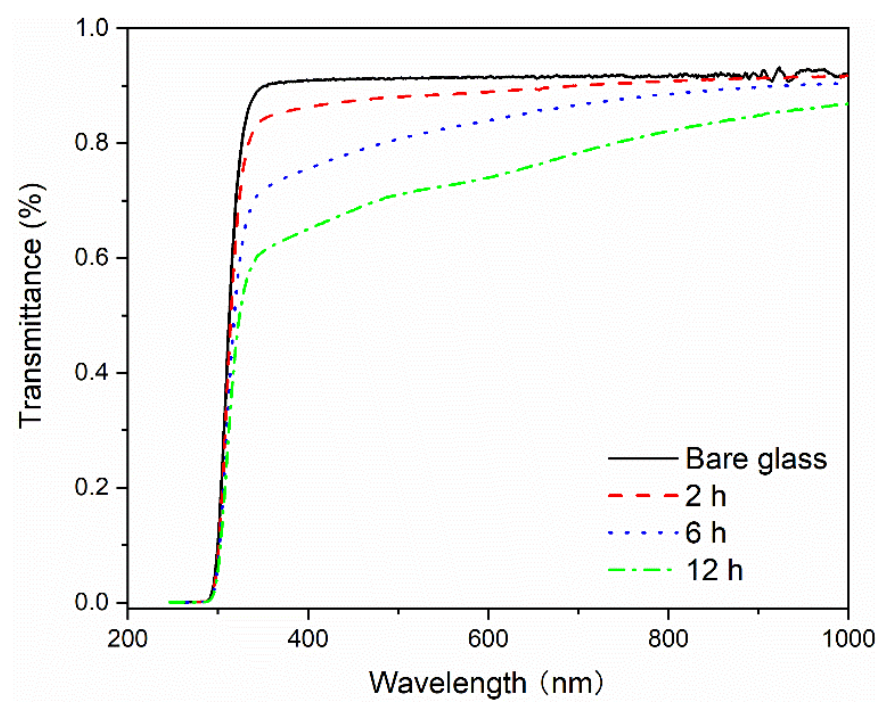

Figure 2 Light transmittance as a function of wavelength for PDA film on glass slide prepared with different deposition times: $2 \mathrm{~h}, 6 \mathrm{~h}$, and $12 \mathrm{~h}$. The representative curve, out of 15 measurements, is provided for each sample. 
composition of DA (N/C $=0.125)$. Next, deconvolution analysis of high-resolution $\mathrm{C} 1 \mathrm{~s}, \mathrm{O} 1 \mathrm{~s}$, and $\mathrm{N} 1 \mathrm{~s}$ peaks was conducted. ${ }^{9,42,43}$ The representative high-resolution spectra of $\mathrm{C} 1 \mathrm{~s}, \mathrm{O} 1 \mathrm{~s}$ and $\mathrm{N} 1 \mathrm{~s}$ regions, obtained for $2 \mathrm{~h}$ sample, are presented in Figure 1 (panel b, c, d). The corresponding data for 6 and $12 \mathrm{~h}$ samples are available in the Supporting Information (Section 1 ). The $\mathrm{C}$ 1s region comprises five peaks representing $\mathrm{CHx}(284.8 \mathrm{eV}), \mathrm{C}-\mathrm{O} / \mathrm{C}-\mathrm{N}(286.3 \mathrm{eV}), \mathrm{C}=\mathrm{O} / \mathrm{C}=\mathrm{N}(287.8 \mathrm{eV}), \mathrm{COOH}$ $(288.8 \mathrm{eV})$ and $\pi-\pi^{*}(291.1 \mathrm{eV})$. The $\mathrm{N} 1 \mathrm{~s}$ region includes three species representing tertiary/aromatic $\left(=\mathrm{NH}_{2}, 398.5 \mathrm{eV}\right)$, secondary (R-NH-R, $399.9 \mathrm{eV})$, and primary $\left(\mathrm{R}-\mathrm{NH}_{2}, 401.7 \mathrm{eV}\right)$ amine groups. The $\mathrm{O}$ 1s region represents three main components, namely $\mathrm{O}-\mathrm{C}(531.3 \mathrm{eV}), \mathrm{O}=\mathrm{C}(532.8 \mathrm{eV})$ and Oads (534.3 eV, adsorbed $\mathrm{H}_{2} \mathrm{O}$ ).

To investigate the effect of deposition time on the surface properties of the coatings, water contact angle measurements were also conducted (Supporting Information, Section 2). In general, the water contact angle increases with the deposition time for PDA coatings (roughly from 50 to $60^{\circ}$ ), which also suggests variations in the surface properties of the coatings.

To examine the light-absorbing behavior of the PDA films, transmission (straight-through) ellipsometry measurements were conducted. Figure 2 displays the intensity of the light transmitted through bare and PDA-coated glass slides as a function of wavelength. It is evident that all the PDA films demonstrate a broad light absorption behavior (i.e., reduce the transmission intensity) in the UV to NIR range, an effect that becomes stronger towards shorter wavelengths. This observation is consistent with the reported extinction coefficient of the polydopamine particles in solution using UVVis spectroscopy. ${ }^{24,25}$ Notably, these studies suggest that lightabsorption by PDA particles enhances with time, meaning that the extinction coefficient spectrum evolves with the oxidative reaction. Thus, optical modeling of the PDA films requires two essential considerations. First, the optical model should account for the complex and broad light-absorbing nature of PDA. Second, depending on the deposition conditions, i.e., deposition time herein, the optical properties of PDA can vary so each sample may require a unique optical model.

Figure 3 demonstrates the ellipsometric $\psi$ and $\Delta$ spectra collected from the PDA films on silica wafer together with the optimized modeling data. A step-by-step construction of the optical model is used to analyse the PDA samples of different deposition times (Supporting Information, Section 3). For $2 \mathrm{~h}$ deposition (Figure 3, left column), the Cauchy model was first utilized to describe the optical dispersion of PDA. Herein, the
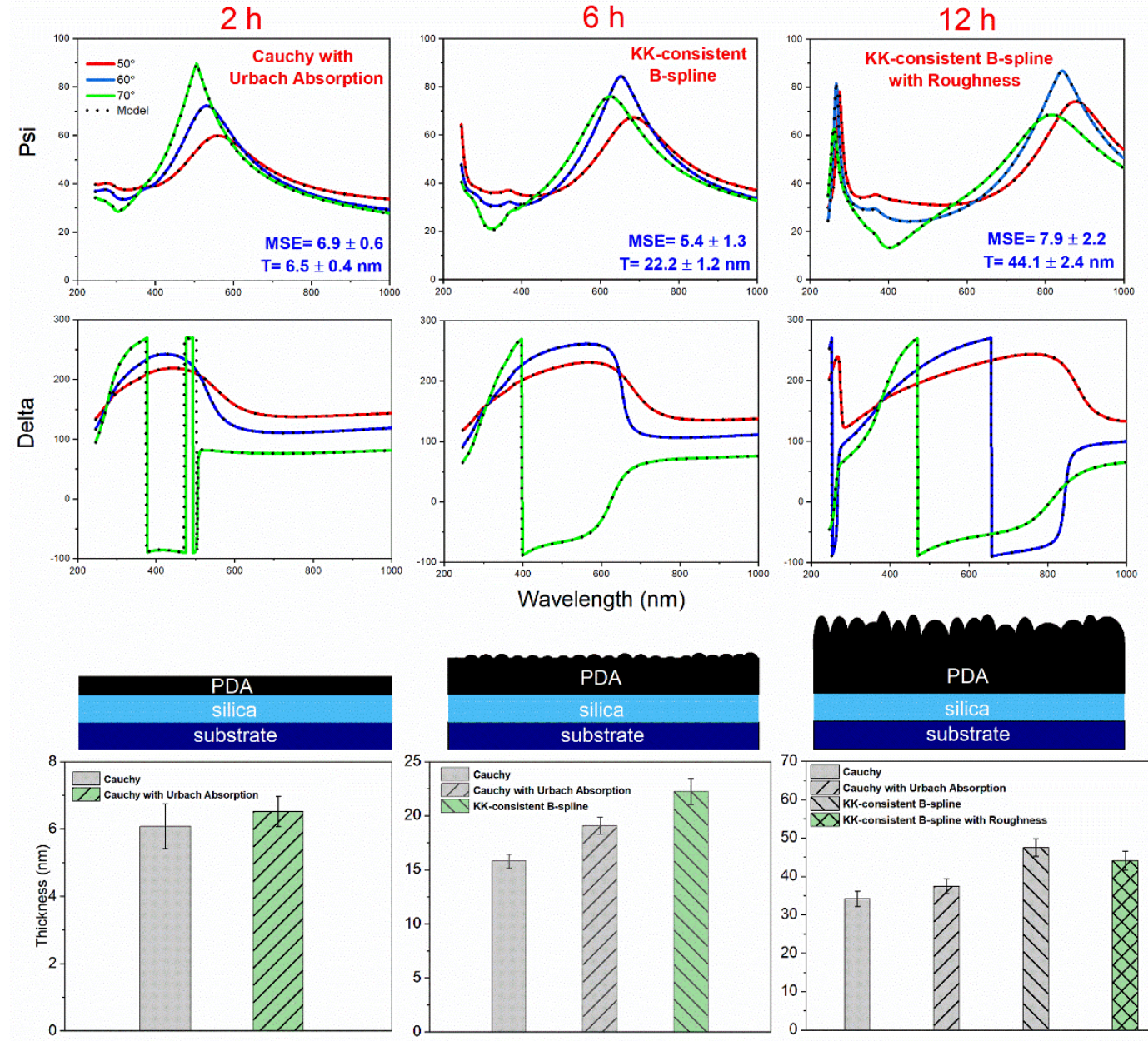

Figure 3 The measured $\psi(\lambda)$ and $\Delta(\lambda)$ data together with the modelled $\psi(\lambda)$ and $\Delta(\lambda)$. Three different optical models are used to describe the optical dispersions of PDA, i.e., Cauchy with Urbach absorption term ( $2 \mathrm{~h}$ ), Kramers-Kronig-consistent B-spline $(6 \mathrm{~h})$, and Kramers-Kronig-consistent B-spline with roughness layer (12 $\mathrm{h})$. The values of mean squared error (MSE) and film thickness (T) are provided for each modeling procedure. Film thickness (bottom row) corresponds to the average of 15 ellipsometric measurements. 
film thickness and A (amplitude parameter) were the free model parameters. It was found that including $B$ and $C$ as free parameters has no significant improvement in the quality of fitting, then constant values of 0.01 and 0 (typical for organic transparent materials) were used. However, the modelled $\psi$ and $\Delta$ spectra poorly matched the experimental data over the entire range of wavelengths (Figure S3, MSE $=37.7$ ). The Cauchy relation is thus not an appropriate model to describe the optical behavior of PDA. Given the light-absorbing nature of PDA, an Urbach absorption term was included to add a small exponentially decaying extinction coefficient to the Cauchy model. Thus, in addition to thickness and $A$, amplitude $(\alpha)$ and exponent $(\beta)$ parameters of the Urbach relation are also free model parameters $\left(E_{b}\right.$ was set to the highest photon energy in the spectrum, i.e., $5 \mathrm{eV}$ ). By doing so, a notable improvement in the fitting quality was achieved (Figure S4, MSE $=6.9 \pm 0.6$ ). For $6 \mathrm{~h}$ deposition (Figure 3, middle column), the Cauchy model, as expected, poorly described the optical properties (Figure S5, MSE = 101.1). Including the Urbach absorption partly improved the fitting quality (Figure S6, MSE = 39.6), yet the modelled data still could not sufficiently match the measured data, e.g., in the $300-400 \mathrm{~nm}$ region. Hence, the Urbach absorption term cannot represent the strong light absorption by PDA obtained by $6 \mathrm{~h}$ deposition. As discussed earlier, the Urbach absorption term is often employed to address minor light absorption in the UV range, whereas it fails to represent large and complex light absorption over a broad range of wavelengths. Two common modeling approaches can be adopted when analysing the ellipsometric data of light-absorbing materials with unknown
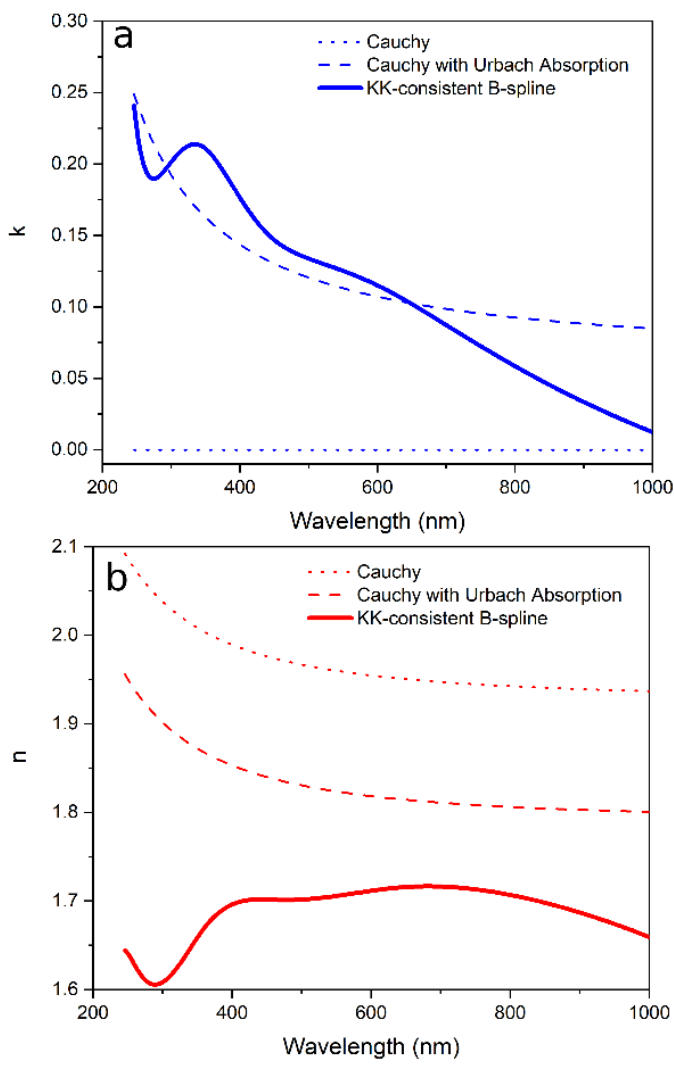

Figure 4 (a)(b) optical dispersions of PDA $6 \mathrm{~h}$ deposition sample obtained from different modeling approaches, (c) effect of number of B-spline nodes on the modelled optical constants of PDA $6 \mathrm{~h}$ deposition sample, (d) two-parameter thickness-roughness uniqueness chart for PDA $12 \mathrm{~h}$ deposition sample

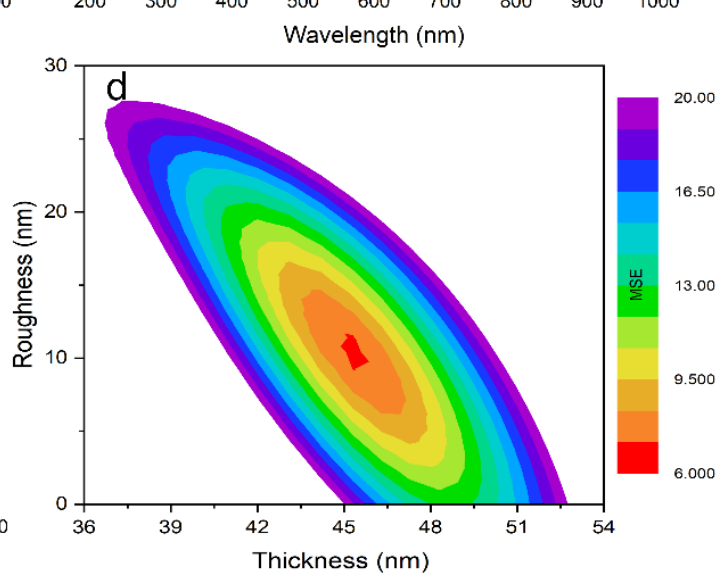

optical properties, i.e., oscillator models and B-spline parametrization. ${ }^{31}$ The oscillator models are used to describe the light absorption at different resonance frequencies using mechanical resonance models, e.g. Gaussian or Tauc-Lorentz oscillators. ${ }^{44,45}$ While B-spline function is not a "physical" model, it can still afford a smooth and continuous dispersion curve with few free parameters. ${ }^{37}$ Considering the complex chemical structure and light-absorbing nature of PDA, we employed Bspline to parametrize the optical constants of PDA and analyse the ellipsometric data. To eliminate the possible non-physical results, only the imaginary part of the dielectric function was parameterized by B-spline, while the real part was calculated using the Kramers-Kronig relation. An optimized resolution of $0.7 \mathrm{eV}$ corresponding to 6 nodes was used. B-spline parametrization of the optical functions of PDA provided a nearly perfect match between the experimental and modelled data over the entire range of wavelengths (Figure S7, MSE = 5.4 \pm 1.3 ). Comparing the three modeling approaches, it is evident that the optical model not only affects the fitting quality and the estimated optical constants, but also greatly influences the estimated thickness value. Accordingly, the estimated film thicknesses are roughly 16, 19 and $22 \mathrm{~nm}$ from Cauchy, Cauchy with Urbach term, and B-spline models, respectively. Therefore, even if the optical dispersions of PDA are not of interest, a wrong dispersion equation can provide an inaccurate estimation of the coating thickness. The underestimated thickness from the Cauchy model can be explained based on the strong correlation between the thickness and optical constant ( $A$ from the Cauchy equation). This can be perceived more

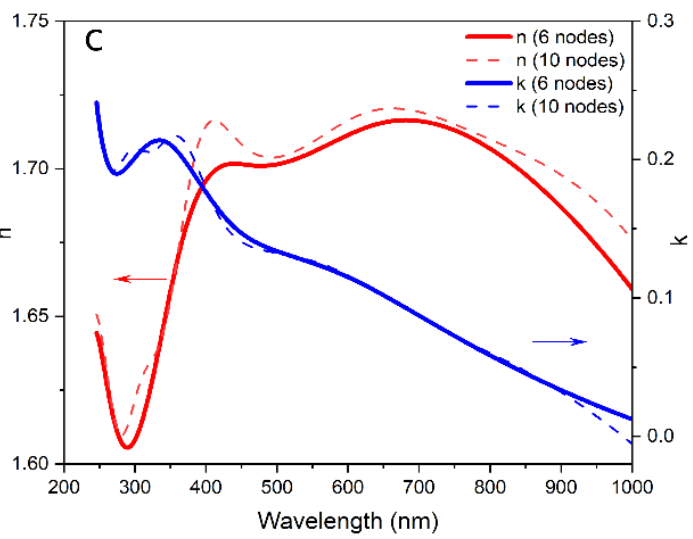


clearly from Figure 4 (panel a, b) where the PDA optical dispersions obtained from the different modeling approaches are compared ( $6 \mathrm{~h}$ deposition). Regarding the Cauchy relation, the obtained dispersion for index of refraction is around 2, which is considerably larger than the typical values for organic materials (roughly between 1.4 to 1.75 ). ${ }^{46}$ This suggests the thickness and optical constants are strongly correlated; hence, an overestimated index of refraction and underestimated optical thickness is found. Addition of the Urbach absorption term leads to a downward shift in the index of refraction dispersion and an extinction coefficient in the range of $0.1-0.25$ (growing with a shift towards the UV range) is found. Regarding B-spline modeling, the index of refraction is shifted further down and a more complex dispersion curve for the extinction coefficient is obtained. In general, the extinction coefficient curve presents complex absorption features and increases when moving to shorter wavelengths reaching a value of around 0.15 to 0.25 in the UV range. The index of refraction shows an anomalous dispersion ( $\mathrm{n}$ decreases towards shorter wavelengths, e.g., in 300-400 $\mathrm{nm}$ ) and varies roughly in the range of 1.6 to 1.7. Determination of the optical dispersions of PDA is a challenge as discussed earlier. A survey through the literature shows that the reported $\mathrm{n}$ and $\mathrm{k}$ for PDA, melanin, and other catecholamine derivatives notably depend on the material preparation and the measurement method. ${ }^{9,47-53}$ For instance, Xiao et al., ${ }^{51}$ Kawamura et al., ${ }^{52}$ and Stavenga et al. ${ }^{53}$ reported $1.7<n<1.8$ for PDA and melanin particles in the 400 $800 \mathrm{~nm}$ region. Vega et al. reported a rather constant value of $\sim$ 1.5 for PDA films in the $300-900 \mathrm{~nm}$ region..$^{48} \mathrm{Li}$ et al. reported $\mathrm{n} \sim 1.7-1.85$ with a dispersion shape similar to our study in the 400-1000 nm region. ${ }^{47}$ Repenko et al. reported a rather constant $\mathrm{n} \sim 1.55$ in the $400-800 \mathrm{~nm}$ region, which increases to around 1.8 in the UV range. Akin et al. reported $n \sim 1.4-1.6$ in the 200-1000 $\mathrm{nm}$ region, which decreases towards shorter wavelengths. ${ }^{50}$ In general, the refractive index of PDA seems to notably depend on its microstructure and particle shape/size and thus the preparation method. On the other hand, the $k$ dispersion in this study and other literature reports are in good agreement both in terms of the shape and the range of values. As discussed regarding B-spline parameterization, one should always minimize the number of nodes (and thus free model parameters) to avoid overparameterization. We performed a thorough sensitivity analysis of the modelled thickness and optical dispersions against the number of nodes (Supporting Information, Section 4). Herein, we briefly discuss how overparameterization can affect the modelling outcome and highlight the importance of finding the minimum number of nodes. Figure 4 (panel c) compares the modelled optical dispersions of the PDA film (6h deposition) using two different numbers of nodes, i.e., 6 and 10 nodes. Accordingly, the number of nodes does not affect the overall shape and values of the optical dispersions. However, using 10 nodes produces small features and peaks in the optical dispersions, which remain unknown to be the real optical behaviour of PDA or merely modelling artefact. For this reason, it is reasonable to choose only 6 nodes that can provide smooth optical dispersions without overfitting.

For $12 \mathrm{~h}$ deposition (Figure 3, right column), both Cauchy (Figure S8, MSE = 149.1) and Cauchy with Urbach term (Figure S9, MSE $=98.5$ ) completely fail to match the experimental data. B-spline parameterization provides a better agreement between the experimental and modeling data (Figure S10, MSE = 12.6), yet the model cannot perfectly match the data for short wavelengths. Considering the rather long deposition time, the PDA film herein is expected to have a relatively rough surface. Hence, an upper roughness layer, modelled as a uniform
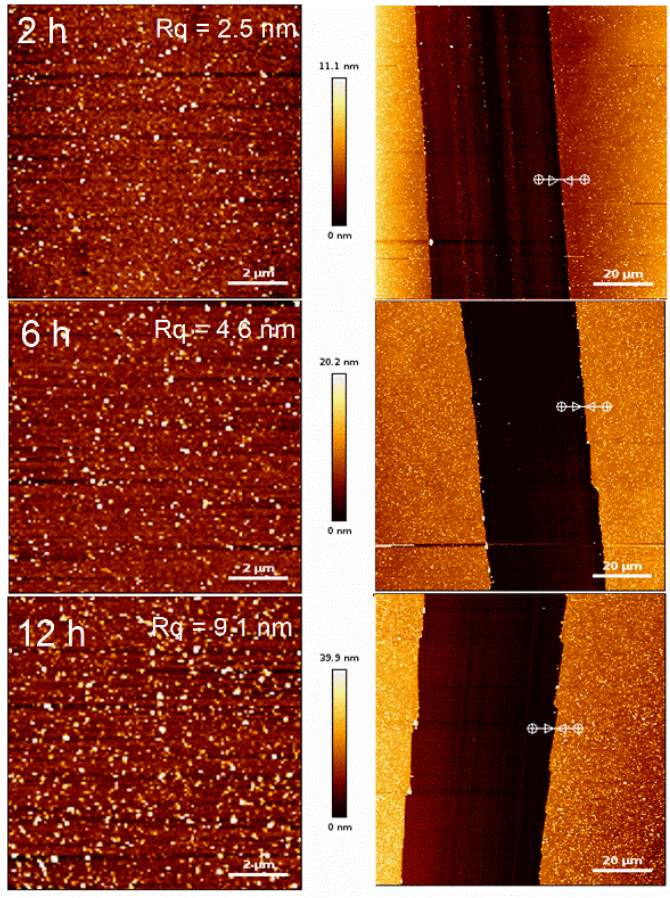
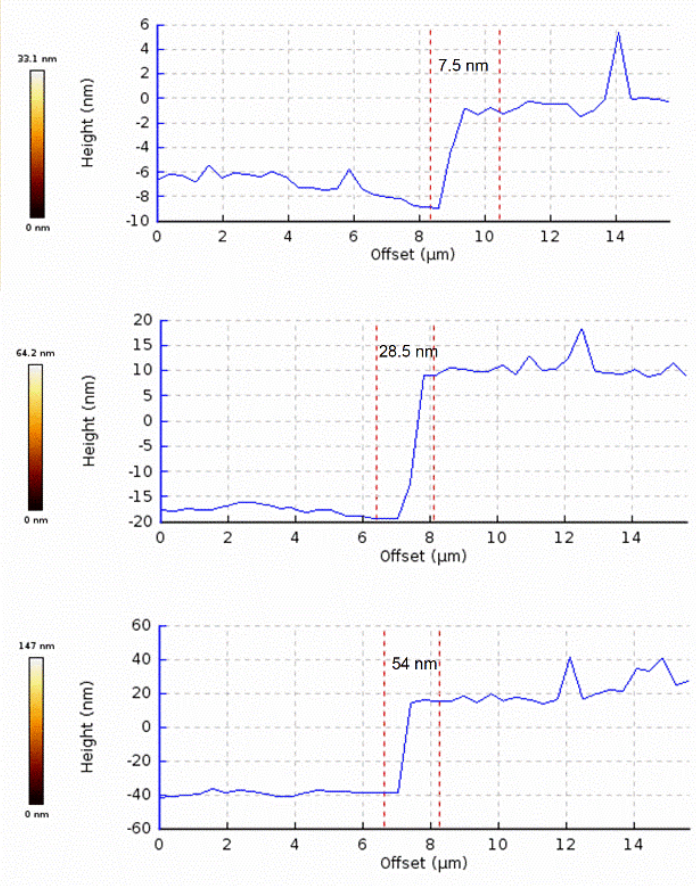

Figure 5 Representative AFM images and corresponding height profiles of PDA films on silicon wafer for $2 \mathrm{~h}$ (top row), $6 \mathrm{~h}$ (middle row) and $12 \mathrm{~h}$ (bottom row) deposition times. 
mixture of PDA-air, is added on top of the B-spline layer. The roughness layer improved the fitting of optical dispersion (Figure S11, MSE $=7.9 \pm 2.2$ ). A roughness value of around $10 \pm$ $2 \mathrm{~nm}$ is estimated from the model. We also conducted a thorough sensitivity analysis of the model parameters for this sample (Supporting Information, Section S4). Herein, we shortly discuss the importance of analysis of the parameters uniqueness, i.e., only one particular combination of the parameters provides a good match between the model and the experimental data. Figure 4 (panel d) depicts the twoparameter uniqueness chart for the PDA film ( $12 \mathrm{~h}$ deposition). Herein, different combinations of thickness-roughness values are characterized by their MSE value. Accordingly, all thicknessroughness combinations provide large MSE values, except a particular combination of thickness $(\sim 45 \mathrm{~nm})$ - roughness ( 10 $\mathrm{nm}$ ) that provides the smallest MSE (red region in the chart). Hence, we can conclude that the modelling outcome is unique. In order to validate the thickness values estimated from ellipsometry, the thickness of the films was also examined using AFM imaging. Figure 5 shows the AFM topography images together with the cross-section height profiles of the scratched area of each PDA film. Overall, the estimated thickness values from the two methods are in agreement (Figure 6), suggesting that the optimized optical models for each PDA sample provided accurate estimates. The AFM data also confirms that the roughness of the coatings increases with the deposition time. It should be noted that the estimated roughness $(12 \mathrm{~h}$ deposition) from ellipsometry is comparable with the calculated $\mathrm{Rq}$ roughness value obtained by AFM. It should be considered, though, that while AFM measurements can supplement the ellipsometry data in terms of constructing appropriate optical models for relatively rough coatings, the roughness values from the two methods are calculated differently and have different mathematical meanings (Supporting Information, Section 5).

We have thus herein demonstrated that PDA films, depending on the preparation condition, can have different optical behavior and microstructure, e.g., surface roughness, which necessitate rigorous modeling analysis and construction of exclusive optical models for each PDA sample. The B-spline parameterization in general provided reliable modelling outcome in terms of the film thickness. In this regard, we also tested if fixing the optical constants (using the values for PDA 6 h) can provide accurate estimates of the film thickness (Figure S12, S13). Regarding the optical properties, we observed that the modelled $\mathrm{k}$ dispersion remains almost the same for all the samples in terms of shape and the range. Contrarily, $n$ dispersion showed dependence on the deposition time, i.e., while the overall shape remains the same, $\mathrm{n}$ increases with deposition time. In this case, we cannot certainly verify if this is due to a variation in the physicochemical properties of PDA (e.g. variations in chemical composition and particle size) or merely modelling artefact. To further investigate this point (Supporting Information, Section S6), we used fixed PDA thickness in the models (using the estimated values from AFM), leaving the optical constants as the model variables. Similarly, it was found that the dispersion shapes are almost independent of deposition time, but $\mathrm{n}$ in particular increases with the

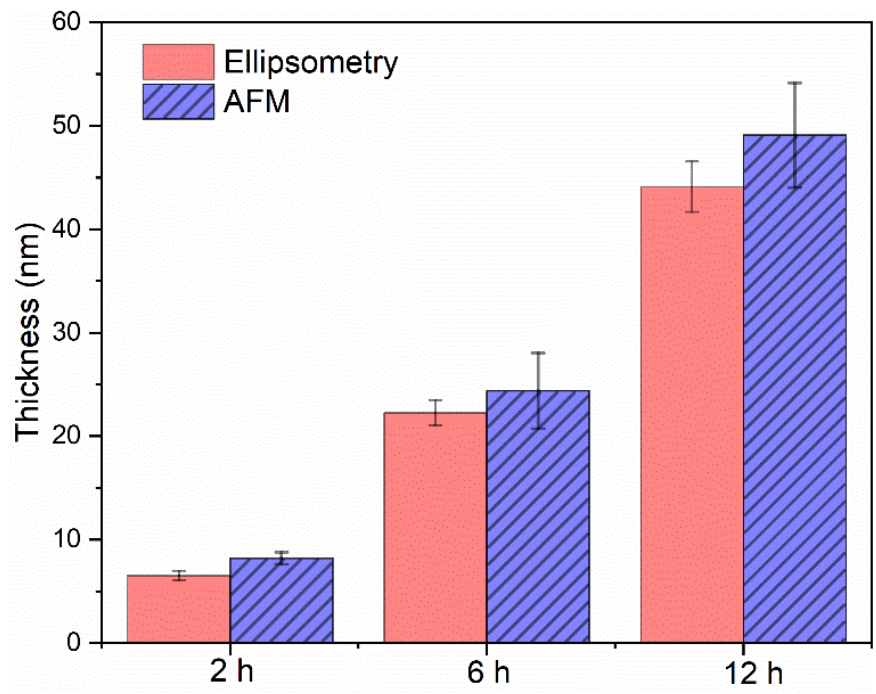

Figure 6 Thickness of PDA films measured with AFM and ellipsometry. For AFM thickness, 30 height profiles ( 3 images, 10 height profiles in each) were examined. The ellipsometry thickness corresponds to 15 measurements.

deposition time. It should be also noted that the investigated samples herein all possessed relatively smooth surfaces (due to a rather short reaction time and multistep preparation method). However, for PDA samples with strong granular surface structure and (or) thickness nonuniformity (relatively long deposition times) ${ }^{10,11}$ one must consider the required nonideality corrections in the optical model. To verify if the step-by-step modeling approach also works for the standard PDA samples, we prepared a thicker and rougher PDA film obtained by $24 \mathrm{~h}$ one-step deposition (Supporting Information, Section S7). It was found that the modelling procedure is useful also for this sample; however, extra consideration was needed to avoid correlation between the parameters due to the uneven surface structure. Last but not least, it should be noted that PDA coatings possess a rather porous and heterogeneous internal structure. ${ }^{11}$ Accordingly, while an isotropic uniform box model is generally used to represent the film when modeling the ellipsometric data, a PDA coating is more like a non-uniform mixture of PDA particles and void, which can also demonstrate gradients of particle size, particle chemistry, and particle volume content in the vertical direction, all of which can affect the optical properties measured by ellipsometry (in particular n). Therefore, the optical dispersions obtained from the B-spline parameterization of the PDA ellipsometric data are more correctly the "pseudo" optical constants rather than the intrinsic properties of the material.

\section{Summary and Conclusion}

In this study, we demonstrated that the light-absorbing nature of PDA is an essential factor that must be considered when assessing the ellipsometric data of such coatings. Accordingly, the commonly but erroneously used Cauchy equation cannot accurately describe the optical behavior of PDA. While addition of the Urbach absorption term to the Cauchy model led to an 
improved modeling quality, the more accurate representation of the optical dispersion was obtained by a KK-consistent Bspline model. Overall, a fallacious optical model not only provides inaccurate and possibly non-physical optical dispersions but also renders incorrect estimation of the film thickness. Besides, an appropriate optical model solely cannot guarantee a decent modeling quality and physically plausible solutions, but extra care is needed for optimizing the number of free parameters and assessing their cross-correlation. Finally, the rough and porous structure of PDA coatings is a factor that may be considered in the optical model.

\section{Conflicts of interest}

There are no conflicts to declare

\section{Acknowledgments}

R. Q. acknowledges a stipend from the China Scholarship Council (CSC). The authors also kindly acknowledge Dr. Thomas Wagner (Quantum Design Europe GmbH, Darmstadt, Germany) for the constructive comments on the data analysis.

\section{Notes and references}

1

H. Lee, S. M. Dellatore, W. M. Miller and P. B. Messersmith, Mussel-Inspired Surface Chemistry for Multifunctional Coatings, Science (80-. )., 2007, 318, 426-430. . Miller, B.D.Freeman, D. R. Paul and C. W. Bielawski, Perspectives on poly(dopamine), Chem. Sci., 2013, 4, 3796-3802. M. E. Lynge, R. Van Der Westen, A. Postma and B. Städler, Polydopamine - A nature-inspired polymer coating for biomedical science, Nanoscale, 2011, 3, 4916-4928.

K. Lim, R. R. Y. Chua, B. Ho, P. A. Tambyah, K. Hadinoto and S. S. J. Leong, Development of a catheter functionalized by a polydopamine peptide coating with antimicrobial and antibiofilm properties, Acta Biomater., 2015, 15, 127-138. H. Gao, Y. Sun, J. Zhou, R. Xu and H. Duan, Mussel-inspired synthesis of polydopamine-functionalized graphene hydrogel as reusable adsorbents for water purification, ACS Appl. Mater. Interfaces, 2013, 5, 425-432. S. H. Ku, J. Ryu, S. K. Hong, H. Lee and C. B. Park, General functionalization route for cell adhesion on non-wetting surfaces, Biomaterials, 2010, 31, 2535-2541.

S. M. Kang, N. S. Hwang, J. Yeom, S. Y. Park, P. B.

Messersmith, I. S. Choi, R. Langer, D. G. Anderson and H. Lee, One-step multipurpose surface functionalization by adhesive catecholamine, Adv. Funct. Mater., 2012, 22, 2949-2955.
Ukraintsev, J. Zemek, A. Kromka and F. Rypaček,

Polydopamine-modified nanocrystalline diamond thin films as a platform for bio-sensing applications, Thin Solid Films, 2013, 543, 180-186.
F. Bernsmann, A. Ponche, C. Ringwald, J. Hemmerle, J. Raya, B. Bechinger, J. C. Voegel, P. Schaaf and V. Ball, Characterization of dopamine-melanin growth on silicon oxide, J. Phys. Chem. C, 2009, 113, 8234-8242.

F. Bernsmann, V. Ball, F. Addiego, A. Ponche, M. Michel, J. J. D. A. Gracio, V. Toniazzo and D. Ruch, Dopamine-melanin film deposition depends on the used oxidant and buffer solution, Langmuir, 2011, 27, 2819-2825.

V. Ball, D. Del Frari, V. Toniazzo and D. Ruch, Kinetics of polydopamine film deposition as a function of $\mathrm{pH}$ and dopamine concentration: Insights in the polydopamine deposition mechanism, J. Colloid Interface Sci., 2012, 386, 366-372.

Y. Liu, W. Z. Qiu, H. C. Yang, Y. C. Qian, X. J. Huang and Z. K. $\mathrm{Xu}$, Polydopamine-assisted deposition of heparin for selective adsorption of low-density lipoprotein, RSC Adv., 2015, 5, 12922-12930.

X. Du, L. Li, J. Li, C. Yang, N. Frenkel, A. Welle, S. Heissler, A. Nefedov, M. Grunze and P. A. Levkin, Uv-triggered dopamine polymerization: Control of polymerization, surface coating, and photopatterning, Adv. Mater., 2014, 26, 8029-8033.

F. Ponzio, J. Barthès, J. Bour, M. Michel, P. Bertani, J. Hemmerlé, M. D'Ischia and V. Ball, Oxidant Control of Polydopamine Surface Chemistry in Acids: A MechanismBased Entry to Superhydrophilic-Superoleophobic Coatings, Chem. Mater., 2016, 28, 4697-4705. Y. Lv, S. J. Yang, Y. Du, H. C. Yang and Z. K. Xu, Codeposition Kinetics of Polydopamine/Polyethyleneimine Coatings: Effects of Solution Composition and Substrate Surface, Langmuir, 2018, 34, 13123-13131.

S. Kasemset, L. Wang, Z. He, D. J. Miller, A. Kirschner, B. D. Freeman and M. M. Sharma, Influence of polydopamine deposition conditions on hydraulic permeability, sieving coefficients, pore size and pore size distribution for a polysulfone ultrafiltration membrane, J. Memb. Sci., 2017, 522, 100-115.

H. Coskun, A. Aljabour, L. Uiberlacker, M. Strobel, S. Hild, C. Cobet, D. Farka, P. Stadler and N. S. Sariciftci, Chemical vapor deposition - based synthesis of conductive polydopamine thin-films, Thin Solid Films, 2018, 645, 320325.

G. Sun, F. Zu, N. Koch, J. Rappich and K. Hinrichs, In Situ Infrared Spectroscopic Monitoring and Characterization of the Growth of Polydopamine (PDA) Films, Phys. Status Solidi Basic Res., 2019, 256, 1800308.

W. Ogieglo, H. Wormeester, K. J. Eichhorn, M. Wessling and N. E. Benes, In situ ellipsometry studies on swelling of thin polymer films: A review, Prog. Polym. Sci., 2015, 42, 42-78.

K. Vedam, Spectroscopic ellipsometry: A historical overview, Thin Solid Films, 1998, 313-314, 1-9.

D. R. Dreyer, D. J. Miller, B. D. Freeman, D. R. Paul and C. W. Bielawski, Elucidating the structure of poly(dopamine), Langmuir, 2012, 28, 6428-6435.

J. Liebscher, R. Mrówczyński, H. A. Scheidt, C. Filip, N. D. Haìdade, R. Turcu, A. Bende and S. Beck, Structure of 
polydopamine: A never-ending story?, Langmuir, 2013, 29, 10539-10548.

T. G. Barclay, H. M. Hegab, S. R. Clarke and M. GinicMarkovic, Adv. Mater. Interfaces, 2017, 4, 1601192. W. Sheng, B. Li, X. Wang, B. Dai, B. Yu, X. Jia and F. Zhou, Brushing up from 'anywhere' under sunlight: $A$ universal surface-initiated polymerization from polydopaminecoated surfaces, Chem. Sci., 2015, 6, 2068-2073.

J. Cui, J. Iturri, J. Paez, Z. Shafiq, C. Serrano, M. d'Ischia and A. del Campo, Dopamine-Based Coatings and Hydrogels:

Toward Substitution-Related Structure-Property Relationships, Macromol. Chem. Phys., 2014, 215, 24032413.

V. Ball, Determination of the extinction coefficient of "polydopamine" films obtained by using $\mathrm{NaIO} 4$ as the oxidant, Mater. Chem. Phys., 2017, 186, 546-551.

F. Bernsmann, O. Ersen, J. C. Voegel, E. Jan, N. A. Kotov and V. Ball, Melanin-Containing Films: Growth from Dopamine Solutions versus Layer-by-Layer Deposition,

ChemPhysChem, 2010, 11, 3299-3305.

28 J. H. Cho, R. Katsumata, S. X. Zhou, C. Bin Kim, A. R. Dulaney, D. W. Janes and C. J. Ellison, Ultrasmooth Polydopamine Modified Surfaces for Block Copolymer Nanopatterning on Flexible Substrates, ACS Appl. Mater. Interfaces, 2016, 8, 7456-7463.

N. Singh, F. Sallem, C. Mirjolet, T. Nury, S. K. Sahoo, N. Millot and R. Kumar, Polydopamine Modified Superparamagnetic Iron Oxide Nanoparticles as Multifunctional Nanocarrier for Targeted Prostate Cancer Treatment, Nanomaterials, 2019, 9, 138. H. Fujiwara, Spectroscopic Ellipsometry: Principles and Applications, John Wiley \& Sons, Ltd, 2007.

H. G. Tompkins and J. N. Hilfiker, Spectroscopic Ellipsometry: Practical Application to Thin Film Characterization, Momentum Press, 2016. H. Fujiwara and R. W. Collins, Spectroscopic Ellipsometry for Photovoltaics, Springer, 2018, vol. 1.

E. Agocs, B. Fodor, B. Pollakowski, B. Beckhoff, A. Nutsch, M. Jank and P. Petrik, Approaches to calculate the dielectric function of $\mathrm{ZnO}$ around the band gap, Thin Solid Films, 2014, 571, 684-688.

S. Schöche, N. Hong, M. Khorasaninejad, A. Ambrosio, E. Orabona, P. Maddalena and F. Capasso, Optical properties of graphene oxide and reduced graphene oxide determined by spectroscopic ellipsometry, Appl. Surf. Sci., 2017, 421, 778-782. Optical constants of graphene measured by spectroscopic ellipsometry, Appl. Phys. Lett., 2010, 97, 091904.

J. W. Weber, T. A. R. Hansen, M. C. M. Van De Sanden and

R. Engeln, B-spline parametrization of the dielectric function applied to spectroscopic ellipsometry on amorphous carbon, J. Appl. Phys., 2009, 106, 123503. B. Johs and J. S. Hale, Dielectric function representation by B-splines, Phys. Status Solidi Appl. Mater. Sci., 2008, 205, 715-719.
Wiley Interdiscip. Rev. Comput. Stat., 2010, 2, 637-653. D. V. Likhachev, Selecting the right number of knots for Bspline parameterization of the dielectric functions in spectroscopic ellipsometry data analysis, Thin Solid Films, 2017, 636, 519-526.

D. E. Aspnes, J. B. Theeten and F. Hottier, Investigation of effective-medium models of microscopic surface roughness by spectroscopic ellipsometry, Phys. Rev. B, 1979, 20, 3292-3302.

D. Lehmann, F. Seidel and D. R. T. Zahn, Thin films with high surface roughness: thickness and dielectric function analysis using spectroscopic ellipsometry, Springerplus, 2014, 3, 82.

S. Rella, E. Mazzotta, A. Caroli, M. De Luca, C. Bucci and C. Malitesta, Investigation of polydopamine coatings by X-ray Photoelectron Spectroscopy as an effective tool for improving biomolecule conjugation, Appl. Surf. Sci., 2018, 447, 31-39.

R. A. Zangmeister, T. A. Morris and M. J. Tarlov, Characterization of polydopamine thin films deposited at short times by autoxidation of dopamine, Langmuir, , DOI:10.1021/la400587j.

F. Wooten, Optical properties of solids, Academic Press, 2013.

D. V. Likhachev, N. Malkova and L. Poslavsky, Modified Tauc-Lorentz dispersion model leading to a more accurate representation of absorption features below the bandgap, Thin Solid Films, 2015, 589, 844-851.

T. Higashihara and M. Ueda, Recent Progress in High Refractive Index Polymers, Macromolecules, 2015, 48 1915-1929.

W. Li, A. Patil, X. Zhou, Z. Wang, M. Xiao, M. D. Shawkey, N. C. Gianneschi and A. Dhinojwala, Characterization of broadband complex refractive index of synthetic melanin coatings and their changes after ultraviolet irradiation, Appl. Phys. Lett., 2020, 117, 203701.

M. Vega, E. M. Martín del Valle, M. Pérez, C. Pecharromán and G. Marcelo, Color Engineering of Silicon Nitride Surfaces to Characterize the Polydopamine Refractive Index, ChemPhysChem, 2018, 19, 3418-3424.

T. Repenko, A. Rix, A. Nedilko, J. Rose, A. Hermann, R. Vinokur, S. Moli, R. Cao-Milàn, M. Mayer, G. von Plessen, A. Fery, L. De Laporte, W. Lederle, D. N. Chigrin and A. J. C. Kuehne, Strong Photoacoustic Signal Enhancement by Coating Gold Nanoparticles with Melanin for Biomedical Imaging, Adv. Funct. Mater., 2018, 28, 1705607. Ü. Akın, í. Çelik, Ç. Avcı, N. Tuğluoğlu and Ö. F. Yüksel, Some optical properties of melanin thick film, Mater. Today Proc., 2019, 18, 1972-1977.

M. Xiao, Y. Li, M. C. Allen, D. D. Deheyn, X. Yue, J. Zhao, N. C. Gianneschi, M. D. Shawkey and A. Dhinojwala, BioInspired Structural Colors Produced via Self-Assembly of Synthetic Melanin Nanoparticles, ACS Nano, 2015, 9, 54545460.

A. Kawamura, M. Kohri, G. Morimoto, Y. Nannichi, T. Taniguchi and K. Kishikawa, Full-Color Biomimetic Photonic Materials with Iridescent and Non-Iridescent Structural 
Journal Name

Colors, Sci. Rep., 2016, 6, 33984

53 D. G. Stavenga, H. L. Leertouwer, D. C. Osorio and B. D. Wilts, High refractive index of melanin in shiny occipital feathers of a bird of paradise, Light Sci. Appl., 2015, 4, e243-e243. 